\title{
Fatigue fracture of tough hydrogels
}

\section{Citation}

Bai, Ruobing, Quansan Yang, Jingda Tang, Xavier P. Morelle, Joost Vlassak, and Zhigang Suo. 2017. "Fatigue Fracture of Tough Hydrogels." Extreme Mechanics Letters 15 (September): 91-96. doi:10.1016/j.eml.2017.07.002.

\section{Published Version}

10.1016/j.eml.2017.07.002

\section{Permanent link}

http://nrs.harvard.edu/urn-3:HUL.InstRepos:34622827

\section{Terms of Use}

This article was downloaded from Harvard University's DASH repository, and is made available under the terms and conditions applicable to Open Access Policy Articles, as set forth at http:// nrs.harvard.edu/urn-3:HUL.InstRepos:dash.current.terms-of-use\#OAP

\section{Share Your Story}

The Harvard community has made this article openly available.

Please share how this access benefits you. Submit a story.

Accessibility 


\title{
Fatigue Fracture of Tough Hydrogels
}

Ruobing Baia, Quansan Yanga, Jingda Tanga,b, Xavier P. Morellea , Joost Vlassak ${ }^{a}$, Zhigang Suo ${ }^{a}$,*

a John A. Paulson School of Engineering and Applied Sciences, Kavli Institute for Bionano Science and Technology, Harvard University, Cambridge, MA 02138, USA

b State Key Lab for Strength and Vibration of Mechanical Structures, Department of Engineering Mechanics, Xi'an Jiaotong University, Xi'an 710049, China

*Corresponding author. E-mail: suo@seas.harvard.edu

\begin{abstract}
:
Tough hydrogels of many chemical compositions have been developed in recent years, but their fatigue fracture has not been studied. The lack of study hinders further development of hydrogels for applications that require long lifetimes under cyclic loads. Examples include tissue engineering, soft robots, and stretchable electronics. Here we study the fatigue fracture of a polyacrylamide-alginate tough hydrogel. We find that the stress-stretch curve changes cycle by cycle, and reaches a steady state after thousands of cycles. The threshold for fatigue fracture is about $53 \mathrm{~J} / \mathrm{m}^{2}$, much below the fracture energy $\left(\sim 10,000 \mathrm{~J} / \mathrm{m}^{2}\right)$ measured under monotonic load. Nonetheless, the extension of crack per cycle in the polyacrylamide-alginate tough hydrogel is much smaller than that in a single-network polyacrylamide hydrogel.
\end{abstract}

Keywords: tough hydrogel, fatigue fracture, polyacrylamide, alginate, shakedown 


\section{Introduction}

Hydrogels are under intense development for applications such as tissue engineering [13], drug delivery [4, 5], and soft machines [6-12]. Hydrogels tend to be soft and brittle, but many applications require hydrogels to sustain appreciable loads and deformation. Several strategies have been developed to synthesize tough hydrogels, including double network hydrogels [13, 14], nano- and micro-composite hydrogels [15-17], as well as tri-block copolymers and hydrophobic associated hydrogels $[18,19]$. The fracture toughness has been enhanced by orders of magnitude, from $10 \mathrm{~J} / \mathrm{m}^{2}$ to $10,000 \mathrm{~J} / \mathrm{m}^{2}$. The mechanical behavior of tough hydrogels has become a field of active research $[20,21]$.

High toughness of these hydrogels is achieved by breaking sacrificial bonds, such as covalent bonds in a short-chain network [13], or ionic crosslinks [14, 18]. Consequently, tough hydrogels are prone to degradation under cyclic loads. Examples include changes in elastic modulus [22], in hysteresis of stress-stretch curves [23, 24], and in functional characteristics of devices [25]. Fatigue fracture of tough hydrogels, however, has remained unexplored. This lack of information on fatigue fracture of tough hydrogels hinders their further development, knowing that fatigue fracture is a critical mode of failure of all other tough materials, including metals, plastics, elastomers and composites [26-34].

We have recently studied fatigue fracture of a polyacrylamide hydrogel, a brittle hydrogel with a single network of covalent crosslinks [35]. Here we explore fatigue fracture of tough hydrogels, which commonly include two interpenetrating networks. We focus on a polyacrylamide-alginate tough hydrogel, where polyacrylamide forms a network of covalent crosslinks, and alginate forms a network of ionic crosslinks [14]. We find that the tough hydrogel exhibits shakedown after prolonged cycling: the stress-stretch curve changes cycle by cycle, and reaches a steady state after thousands of cycles. The threshold for fatigue fracture is much below the toughness under monotonic load, but the extension of crack per cycle in the polyacrylamidealginate tough hydrogel is much smaller than that in a single-network polyacrylamide hydrogel. 


\section{Experimental section}

\subsection{Preparation of tough hydrogel samples}

We purchased from Sigma Aldrich the following substances: acrylamide (AAm, monomer), N,N'-methylenebis(acrylamide) (MBAA, crosslinker), N,N,N',N'tetramethylethylenediamine (TEMED, accelerator), ammonium persulfate (APS, initiator), and calcium chloride. We purchased from FMC Biopolymer the sodium alginate (Manugel GMB).

We prepared polyacrylamide-alginate tough hydrogels following the two-step method described in [36].

In the first step, a single-network polyacrylamide hydrogel with interpenetrating sodium alginate was prepared. $40.54 \mathrm{~g}$ AAm and $6.76 \mathrm{~g}$ sodium alginate were dissolved in $300 \mathrm{ml}$ deionized water to form an aqueous solution. We then added MBAA, TEMED and APS in quantities of $0.0012,0.0025$ and 0.0042 times the weight of AAm. The pre-gel solution was then degassed and injected into a $50 \times 50 \times 1.65 \mathrm{~mm}^{3}$ plastic mold and covered with a glass plate. The sample was stored at room temperature for 24 hours to form the polyacrylamide network. The sodium-alginate chains are believed to interpenetrate the polyacrylamide network, but do not form a network [36].

In the second step, the prepared hydrogel was soaked in a $0.5 \mathrm{M}$ calcium chloride solution for at least 2 days. This allows sufficient time for the exchange of calcium sodium ions between the hydrogel and the external solution, so that an alginate network of calcium crosslinks forms. To ensure that the concentration of calcium ions in the external solution keeps nearly constant before and after soaking the hydrogel, we prepared the calcium chloride solution with a weight at least 10 times the weight of the hydrogel. After soaking, the hydrogel was taken out of the solution and tested. The average thickness of the samples after soaking was measured to be $2.1 \mathrm{~mm}$. 
We also prepared a single-network polyacrylamide hydrogel following the method in the first step, but without adding the sodium alginate.

\subsection{Testing method}

We studied the stress-stretch behavior and fatigue fracture using the pure shear test [14, 29] (Fig. 1a). A thin sheet of sample, of a long rectangular shape $\left(10 \times 50 \times 2.1 \mathrm{~mm}^{3}\right)$, was fixed to two rigid grips, and mounted in a tensile tester (Instron model 5966) with a $500 \mathrm{~N}$ load cell.

To minimize dehydration during the test, we made an acrylic chamber and sealed it around the sample with petrolatum (Fig. 1a \& Fig. 3a). Water droplets were sprayed on the inner surface of the chamber to maintain the humidity. All the samples were weighed before and after the test, and no more than $3 \%$ weight was lost.

During the test, the tensile tester pulled the distance between the two grips cyclically between $H$ and $\lambda H$, where $\lambda$ defines the maximum applied stretch per unit cycle (Fig. $1 \mathrm{~b} \&$ Fig. 3b). We recorded the force-displacement curve of each unnotched sample over many cycles, calculated the nominal stress (i.e., the applied force divided by the cross-sectional area in the undeformed state), and plotted the nominal stress as a function of stretch (Fig. 1c\&d). For the fatigue fracture tests, we notched a sample with a $20 \mathrm{~mm}$ crack before each test. We marked ruler lines on the surface of the sample, and glued graph papers to the grips (Fig. 3c). As the tensile tester pulled the sample cyclically, a digital camera (Nikon D5200) recorded the extension of the crack. 
(a)

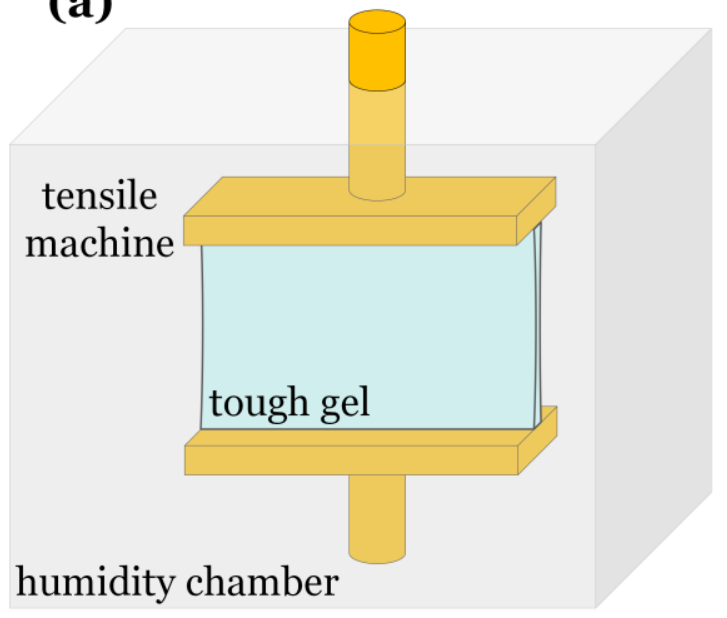

(c)

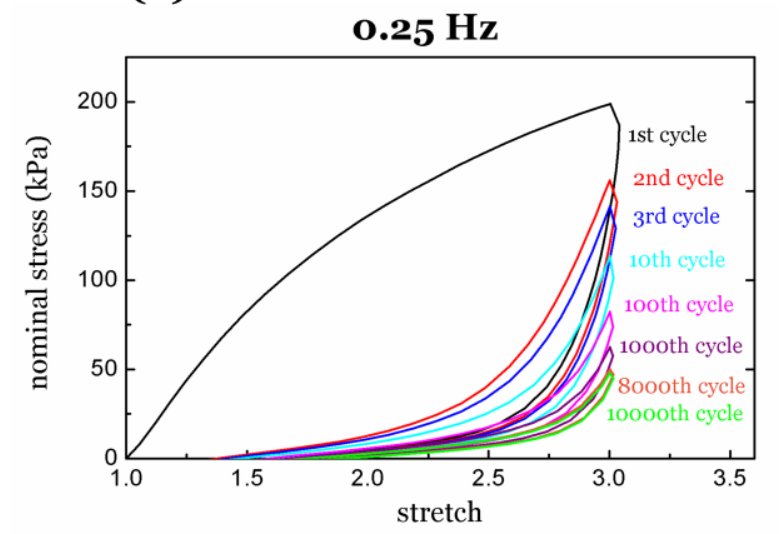

(b)

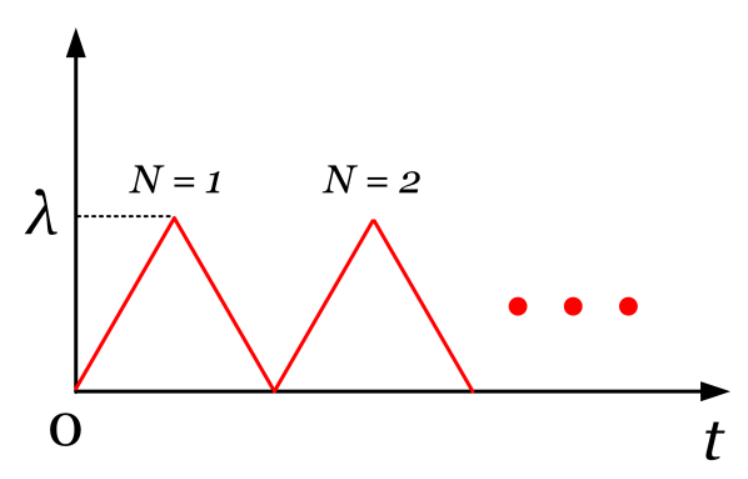

(d)

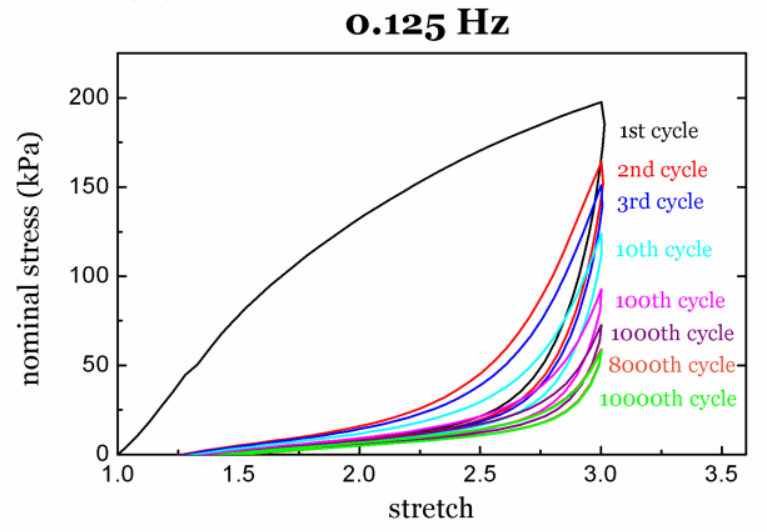

Fig. 1 Shakedown after prolonged cycling. (a) A sample of tough hydrogel is cyclically stretched in the pure shear test. (b) The loading profile of the test. Shakedown of stress-stretch curves was observed in tests with different loading frequencies, and shows no significant rate dependence: The loading frequency is (c) $0.25 \mathrm{~Hz}$, (d) $0.125 \mathrm{~Hz}$.

\section{Shakedown after prolonged cycling}

The stress-stretch curve of a hydrogel or an elastomer often depends on loading history due to various inelastic processes such as the Mullins effect [37, 38], viscoelasticity [39-43], poroelasticity [42-46] and viscoplasticity [47]. In particular, the Mullins effect has been considered as the major factor to explain the softening of double network hydrogels over consecutive loading cycles [48-54], and has been attributed to progressive damage of the polymer networks $[50,55]$. All these studies, however, have been focused on the initial several 
loading cycles. Here we focus on the evolution of the stress-stretch curve over many cycles, and show that the evolution markedly affects the fatigue fracture.

We observe shakedown after prolonged cycling (Fig. 1c\&d). Most of the energy dissipation, material softening and residual strain occur in the first cycle. Afterwards, the stressstretch curve keeps changing, but the changes become smaller and smaller with increasing cycles and become negligible after some large number of cycles. For example, the stress-stretch curves are almost identical for the $8000^{\text {th }}$ and $10,000^{\text {th }}$ cycle. When the stress-stretch curve stops changing, we say that the tough hydrogel has shaken down to a steady state. The maximum stress in the steady state is much lower than that in the first loading cycle (Fig. 2a). A relatively large residual strain was observed after the test.

Shakedown has been extensively studied in ductile metals [26]. During cycling, a metal deforms plastically, and gradually approaches a steady state, when the residual stress generated during the early cycles inhibits further plastic deformation. Further cycling causes reversible elastic response. For the tough hydrogel, we interpret the shakedown as a result of cumulative unzipping of the ionic crosslinks. The polyacrylamide-alginate tough hydrogel consists of interpenetrating polyacrylamide network of covalent crosslinks and alginate network of calcium crosslinks. Upon stretching during the first cycle, some of the calcium crosslinks unzip, leading to softening. The re-zipping of the calcium crosslinks takes hours to days [14], and does not occur within the time period of each cycle. As the sample undergoes more cycles, more calcium crosslinks progressively unzip during each cycle, leading to further softening and accumulated residual strain. The stress-stretch curve reaches a steady state when the cyclic stretch cannot further unzip calcium crosslinks.

The rate-dependent stress-stretch behavior of polyacrylamide-alginate tough hydrogels has been studied recently [54]. However, the required change of loading rate to observe clear rate-dependency is much larger than the range of loading rate in all current experiments. Therefore, within the range of testing period we applied, we anticipate the stress-stretch 
behavior to be almost independent of the loading rate. To show this, we conducted cyclic tests on samples with different cyclic frequencies. No significant differences were observed in the stress-stretch curves (Fig. 1c\&d). As a result, we use a constant loading frequency in all of our cyclic tests, and neglect the rate-dependent effects such as viscoelasticity and poroelasticity.

The changing stress-stretch curves make it hard to quantify fatigue fracture before the steady state. To simplify the problem, we focus on the steady-state fatigue fracture of tough hydrogels in the following discussion. We define the steady state after 2000 loading cycles, when the change of maximum stress becomes relatively small (Fig. 2a). We then plot the associated stress-stretch curves for different maximum stretches in the steady state (Fig. 2b). At 2000 cycles, the stress-stretch curves still exhibit hysteresis, but the hysteresis loops are much narrower than those in the beginning cycles.

(a)

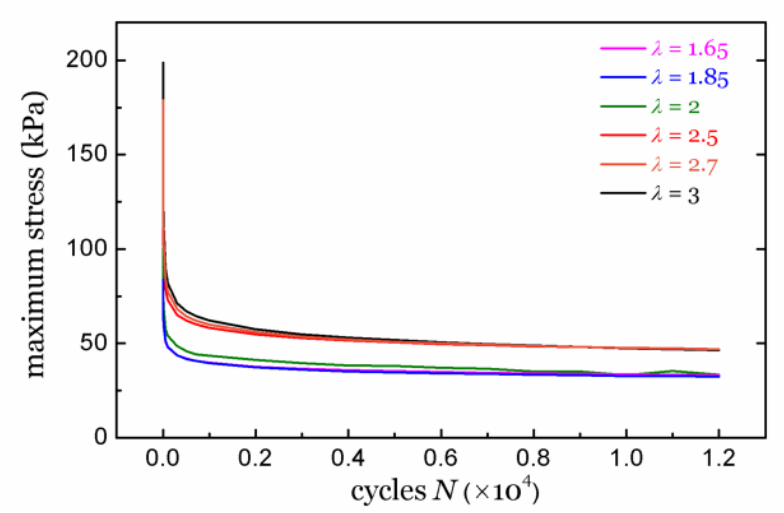

(b)

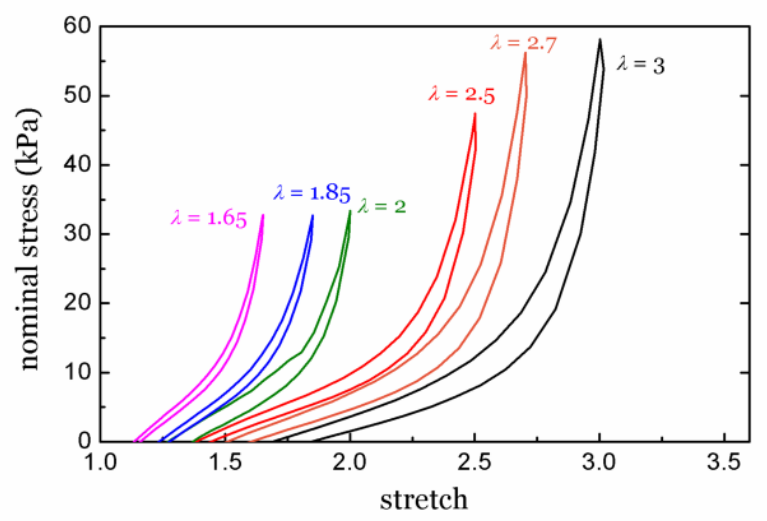

Fig. 2 (a) The maximum stress in each cycle decreases and approaches a steady state. (b) The stress-stretch curves after 2000 cycles for different maximum stretches $\lambda$.

\section{Fatigue fracture of tough hydrogels}

As described in Section 2, fatigue fracture tests were conducted on samples pre-notched with a $20 \mathrm{~mm}$ crack (Fig. 3a). Following the previous discussion, all tests were conducted using the same frequency of $0.25 \mathrm{~Hz}$. The number of loading cycles $N$ was determined from the 
recording time of the camera and the period of one cycle, $4 \mathrm{~s}$ in this case. The crack growth $\Delta c$ is plotted as a function of the number of cycles $N$ (Fig. 4). In all tests, the crack grows rapidly at first, becomes progressively slower afterwards, and in the end reaches a steady state. In the steady state, $\Delta c$ is approximately a linear function of $N$.

The fatigue fracture behavior is related to shakedown. Before reaching the steady state, the stress-stretch curve changes, and the hydrogel softens cycle by cycle. Under the same maximum stretch per cycle, $\lambda$, the maximum load applied to the sample becomes smaller and smaller. As a result, the crack grows fast in the beginning cycles, but gradually slows down. When the stress-stretch curve reaches the steady state, the crack grows linearly with the number of cycles.

\section{(a)}

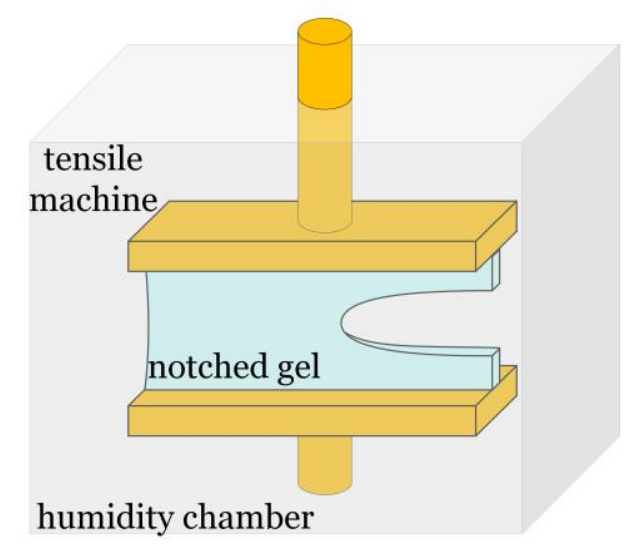

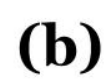

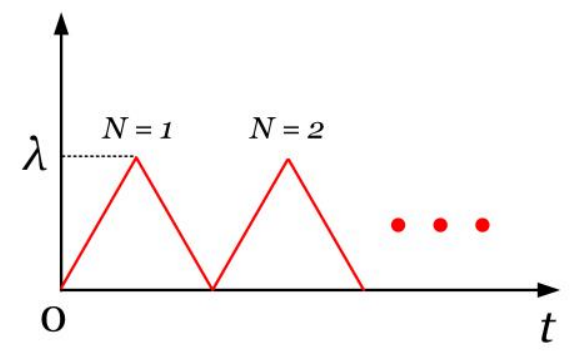

\section{(c)}
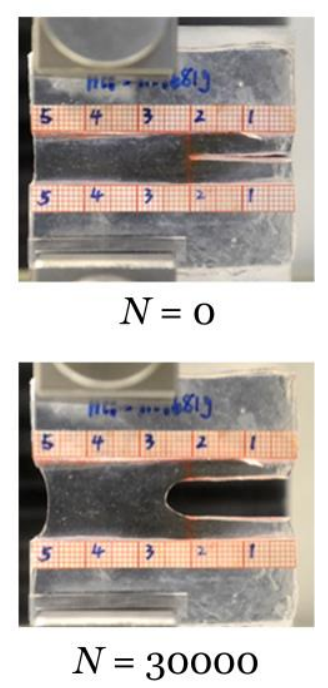

Fig. 3 Fatigue fracture. (a) A notched sample is cyclically stretched. The sample and the grips are enclosed in a chamber with water droplets on its inner surface to control the humidity of the environment. (b) The applied loading profile over cycles. (c) The crack propagates cycle by cycle, and the evolution is recorded by photos. The crack propagation can be measured from the metric papers on the grips. 


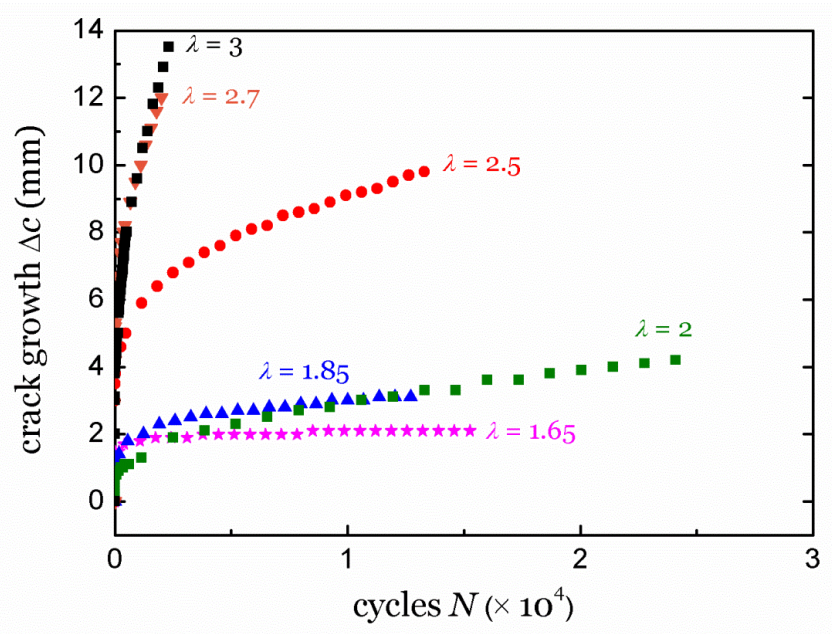

Fig. 4 Crack growth as a function of the number of cycles $N$, under different maximum stretches. The crack growth reaches the steady state after thousands of cycles, and becomes approximately a linear function of $N$.

\section{Characterization of fatigue fracture}

In the tests, we prescribed the maximum stretch $\lambda$ in each cycle. However, to compare experimental data obtained from samples of various materials and geometries, we report the amplitude of load in terms of the energy release rate $G$ calculated from [29]

$$
G=W(\lambda) H,
$$

where $H$ is the distance between the two grips when the sample is undeformed, and $W(\lambda)$ is the elastic energy per unit volume in the undeformed state determined from an unnotched sample. The tough hydrogel is not elastic, and we determine $W(\lambda)$ as follows.

Because a sample shakes down after prolonged cycling, the stress-stretch curve will keep changing until it reaches the steady state. To characterize fatigue fracture in the steady state, we used the stress-stretch curve measured in the steady state, rather than that measured in the first cycle. This method has been previously applied in the study of fatigue fracture of rubbers [37, 56]. To calculate the energy release rate under different applied stretch $\lambda$, we paired the stressstretch curves and the crack growth curves with the same $\lambda$. The area under the stress-stretch curve gives the strain energy density $W(\lambda)$ (Fig. 5a). 
Even after thousands of cycles, the stress-stretch curves still exhibit hysteresis. In the literature on fatigue fracture in elastomer, some authors calculated $W(\lambda)$ by integrating the loading part of the curve [30], while others calculated $W(\lambda)$ by integrating the unloading part of the curve [56, 57]. Following Thomas [30], here we use the loading part of the stress-stretch curve to calculate $W(\lambda)$. Whether the loading or the unloading part is more proper needs further discussion, and is out of the scope of the current paper. In a steady state, the hysteresis loop becomes narrow, and the difference between the energy release rates calculated from the loading and the unloading curves is typically less than $30 \%$.

We then fit the crack growth $\Delta c$ in the steady state as a linear function of $N$, and obtain the extension per cycle $\mathrm{d} c / \mathrm{d} N$ as a function of $G$ (Fig. $5 \mathrm{~b}$ ). When $G$ is relatively small, the extension of crack per cycle $\mathrm{d} c / \mathrm{d} N$ varies almost linearly with $G$. As $G$ increases, $\mathrm{d} c / \mathrm{d} N$ increases steeply, becoming a higher order polynomial function of $G$. Such behavior is similar to the fatigue fracture of elastomers [30, 31, 34].

We fit the linear relation and find its intercept with the $G$ axis, at a finite value of about 53.2 $\mathrm{J} / \mathrm{m}^{2}$, which we define as the threshold for fatigue fracture, denoted by $\Gamma_{0}$. Below this value, no crack growth was observed in the steady state until the end of the experiment.

In the fatigue fracture of elastomers, it has been shown experimentally that different geometries of the specimens lead to the same curve of $\mathrm{d} c / \mathrm{d} N$ vs. $G$ [30]. We have not verified this point experimentally for the tough hydrogel, but also anticipate the curve for the gel to be independent of specimen geometry. Furthermore, it was shown previously that fracture energy of the tough hydrogel measured under the monotonic stretch is insensitive to the size and shape of the specimens [14]. 

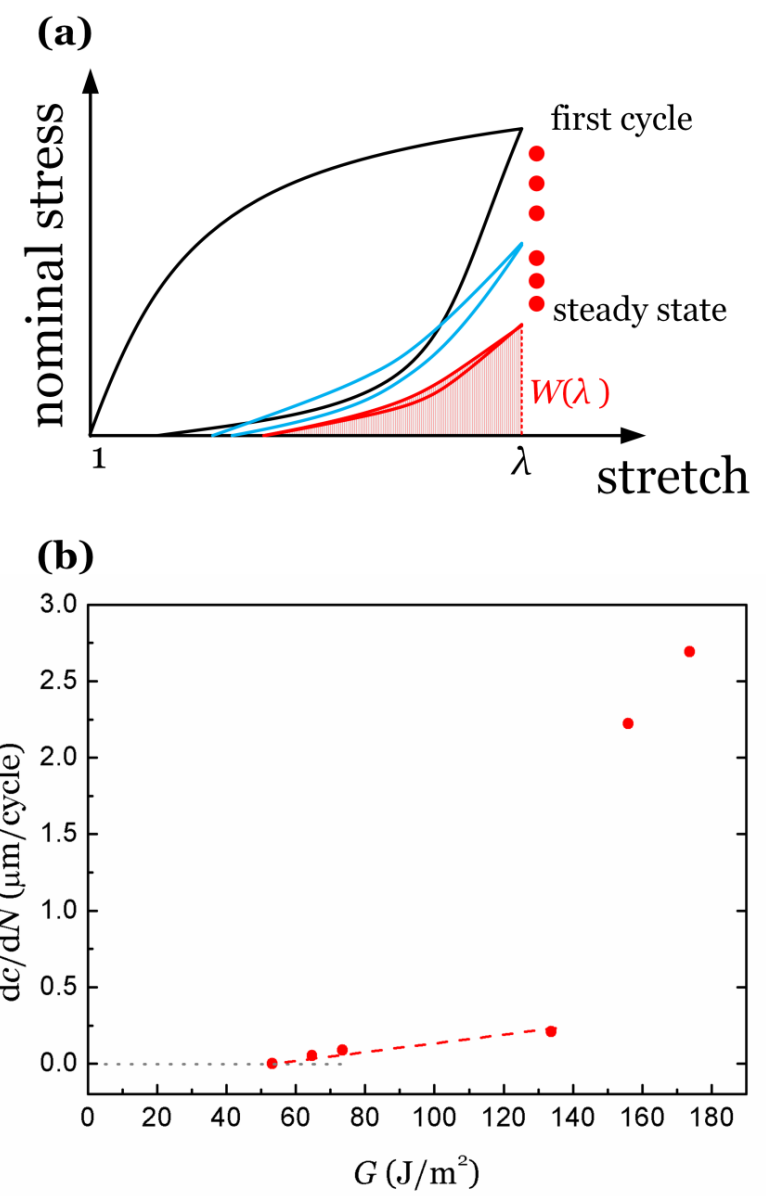

Fig. 5 (a) The energy release rate is calculated by integrating the stress-stretch curve in the steady state of the material far ahead of the crack tip. (b) In the steady state, the extension of crack per cycle, $\mathrm{d} c / \mathrm{d} N$, is plotted as a function of energy release rate $G$. A threshold is found, below which no crack propagation in the steady state occurs. Close above the threshold, $\mathrm{d} c / \mathrm{d} N$ is found as a linear function of $G$. $\mathrm{d} c / \mathrm{d} N$ then increases rapidly with $G$.

\section{Discussion}

\subsection{Polyacrylamide hydrogels show no "shakedown"}

We now compare the polyacrylamide-alginate tough hydrogel with a polyacrylamide hydrogel. The polyacrylamide hydrogel has a single network of covalent crosslinks, and we do not expect shakedown over a large number of cycles. To demonstrate this, we made a polyacrylamide hydrogel of the same size and loaded it under the same condition as the tough hydrogel. To minimize the effect caused by the difference of chemical network between the two gels, we used the same concentration of acrylamide monomer solution and added the same amount of crosslinker. Over 4000 cycles, the stress-stretch curve of the polyacrylamide hydrogel 
remains almost unchanged (Fig. 6a), and the maximum stress in each cycle is constant over the whole period (Fig. 6b). The sample ruptured afterwards due to the brittleness of the hydrogel.

The observation that the polyacrylamide hydrogel maintains the same stress-stretch loop during cyclic loading again confirms that the shakedown in the tough hydrogel is caused by the unzipping of the ionic crosslinks. With no ionic crosslinks, the polyacrylamide hydrogel has stable stress-stretch curves.

(a)

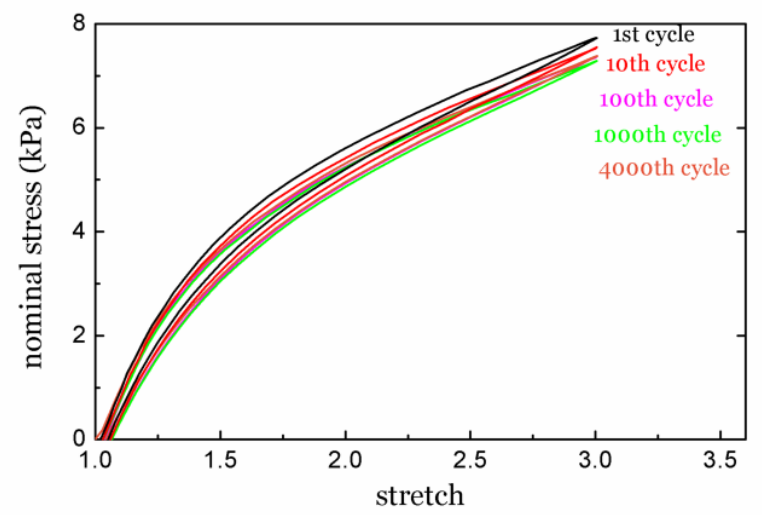

(b)

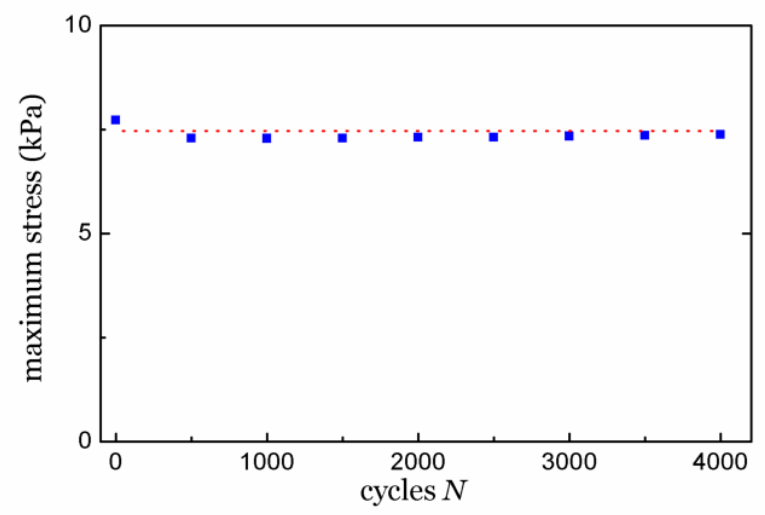

Fig. 6 (a) A polyacrylamide hydrogel maintains stable stress-stretch loops over thousands of cycles. (b) The maximum stress in each cycle remains nearly constant over cycles.

\subsection{Comparison between polyacrylamide hydrogel and tough hydrogel in fatigue fracture}

The fracture toughness of the tough hydrogel is several thousands of $\mathrm{J} / \mathrm{m}^{2}$, much higher than the fracture toughness of the polyacrylamide hydrogel, which is usually on the order of 100 $\mathrm{J} / \mathrm{m}^{2}[14]$. Here we compare their fatigue behavior.

We use the data of fatigue fracture in the polyacrylamide hydrogel from our previous paper [35], and plot it with the data of the tough hydrogel of the current paper. It is shown that even under larger cyclic stretch $\lambda$ and higher mechanical load $G$, after the same number of 
loading cycles, the crack growth in the tough hydrogel is much smaller than the crack growth in the polyacrylamide hydrogel (Fig. 7a).

We quantify the fatigue fracture resistance of the two gels through two parameters: the threshold for fatigue fracture $\Gamma_{0}$, and the slope of $\mathrm{d} c / \mathrm{d} N$ as a function of $G$. The two parameters represent different measures of the fatigue fracture resistance of a material. The threshold $\Gamma_{0}$ defines the critical energy release rate above which fatigue fracture occurs. Thus, a material is more fatigue fracture resistant with a higher threshold $\Gamma_{0}$. When a hydrogel is loaded above the threshold, the slope of dc/dN vs. $G$ determines how fast the crack grows over the loading cycles. The smaller the slope, the better the fatigue fracture resistance. Our preliminary data shows that the tough hydrogel has a better fatigue fracture resistance than the polyacrylamide hydrogel in terms of both parameters (Fig. 7b).

(a)

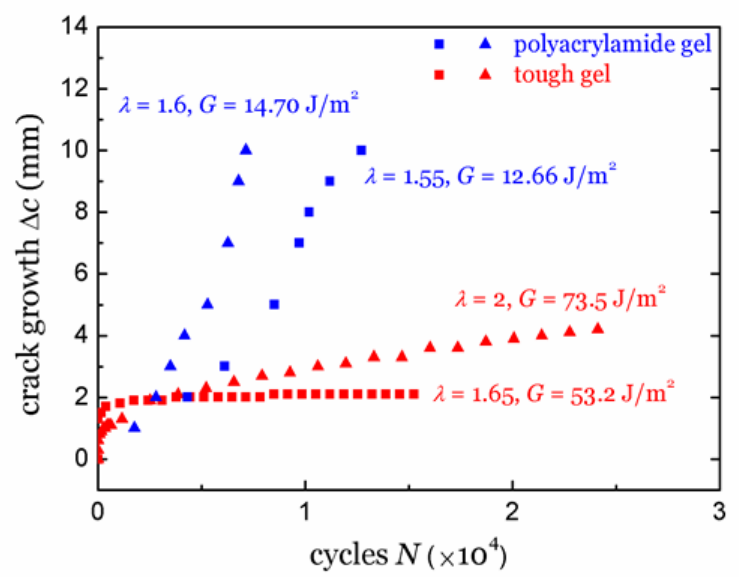

(b)

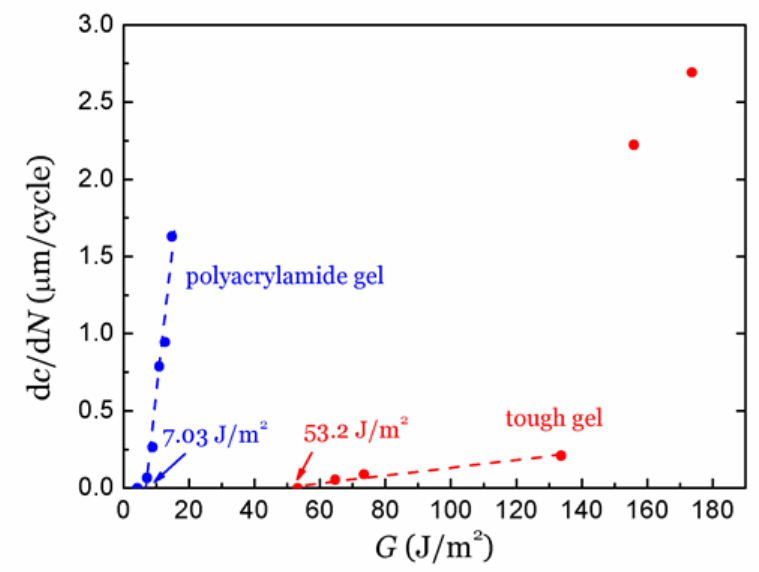

Fig. 7 Comparison between a polyacrylamide hydrogel and a tough hydrogel in fatigue fracture. (a) The crack growth of the polyacrylamide hydrogel is faster (steeper slope) than that of the tough hydrogel, even under a less critical condition (smaller $\lambda$ and $G$ per cycle). (b) The fatigue threshold of the tough hydrogel is higher than that of the polyacrylamide hydrogel. The slope of $\mathrm{dc} / \mathrm{d} N$ vs. $G$ in the tough hydrogel is much lower than that in the polyacrylamide hydrogel. Both factors show that tough hydrogels have better fatigue fracture resistance than polyacrylamide hydrogels.

\section{Conclusion}


The stress-stretch curve of the polyacrylamide-alginate tough hydrogel keeps changing during cyclic loading and reaches a steady state after thousands of cycles. We call this phenomenon shakedown after prolonged cycling. Cycling softens the gel markedly. We measured the crack growth per cycle as a function of the applied mechanical load, quantified by the energy release rate in the steady state. The fatigue threshold of the tough hydrogel is about $53 \mathrm{~J} / \mathrm{m}^{2}$, whereas the fracture toughness of the tough hydrogel under monotonic load easily reaches several thousands of $\mathrm{J} / \mathrm{m}^{2}$. Still, the fatigue crack growth in the tough hydrogel is much slower than that in the polyacrylamide hydrogel. We hope that fatigue fracture of other tough hydrogels will soon be studied and compared. Such studies will aid the development of tough and fatigue-resistant hydrogels.

\section{Acknowledgments}

This work was supported by MRSEC (DMR-14-20570), and by NSF (CMMI-1404653). J. T. was supported by China Scholarship Council (201306010164) as a visiting scholar for two years at Harvard University. Q. Y. was supported by a scholarship from Tsinghua University as a visiting scholar for six months at Harvard University. X. P. M. was supported by the CabeauxJacobs BAEF fellowship for 1 year at Harvard University. We thank Professor David Mooney for the use of the Instron tensile tester.

\section{References}

1. Hassan CM \& Peppas NA (2000) Structure and applications of poly (vinyl alcohol) hydrogels produced by conventional crosslinking or by freezing/thawing methods. Biopolymers. PVA Hydrogels, Anionic Polymerisation Nanocomposites, (Springer), pp 37-65.

2. Lee KY \& Mooney DJ (2001) Hydrogels for tissue engineering. Chemical reviews 101(7):1869-1880.

3. Lienemann PS, Lutolf MP, \& Ehrbar M (2012) Biomimetic hydrogels for controlled biomolecule delivery to augment bone regeneration. Advanced drug delivery reviews 64(12):1078-1089.

4. Langer R (1998) Drug deliveryand targeting. Nature 392(6679):5-10. 
5. Ferreira P, Almeida J, Coelho J, \& Gil M (2011) Photocrosslinkable polymers for biomedical applications (INTECH Open Access Publisher).

6. Keplinger C, et al. (2013) Stretchable, transparent, ionic conductors. Science 341(6149):984-987.

7. Sun JY, Keplinger C, Whitesides GM, \& Suo Z (2014) Ionic skin. Advanced Materials 26(45):7608-7614.

8. Buenger D, Topuz F, \& Groll J (2012) Hydrogels in sensing applications. Progress in Polymer Science 37(12):1678-1719.

9. $\quad$ Yang CH, et al. (2015) Ionic cable. Extreme Mechanics Letters 3:59-65.

10. Yang CH, Chen B, Zhou J, Chen YM, \& Suo Z (2015) Electroluminescence of Giant Stretchability. Advanced Materials.

11. Larson C, et al. (2016) Highly stretchable electroluminescent skin for optical signaling and tactile sensing. Science 351(6277):1071-1074.

12. Beebe DJ, et al. (2000) Functional hydrogel structures for autonomous flow control inside microfluidic channels. Nature 404(6778):588-590.

13. Gong JP, Katsuyama Y, Kurokawa T, \& Osada Y (2003) Double - network hydrogels with extremely high mechanical strength. Advanced Materials 15(14):1155-1158.

14. Sun J-Y, et al. (2012) Highly stretchable and tough hydrogels. Nature 489(7414):133-136.

15. Haraguchi K \& Takehisa T (2002) Nanocomposite hydrogels: a unique organic-inorganic network structure with extraordinary mechanical, optical, and swelling/de-swelling properties. Advanced Materials 14(16):1120.

16. Huang $\mathrm{T}$, et al. (2007) A novel hydrogel with high mechanical strength: a macromolecular microsphere composite hydrogel. Advanced Materials 19(12):1622-1626.

17. Tang J, Chen X, Pei Y, \& Fang D (2016) Pseudoelasticity and Nonideal Mullins Effect of Nanocomposite Hydrogels. Journal of Applied Mechanics 83(11):111010.

18. Henderson KJ, Zhou TC, Otim KJ, \& Shull KR (2010) Ionically cross-linked triblock copolymer hydrogels with high strength. Macromolecules 43(14):6193-6201.

19. Abdurrahmanoglu S, Cilingir M, \& Okay O (2011) Dodecyl methacrylate as a crosslinker in the preparation of tough polyacrylamide hydrogels. Polymer 52(3):694-699.

20. Zhao X (2014) Multi-scale multi-mechanism design of tough hydrogels: building dissipation into stretchy networks. Soft Matter 10(5):672-687.

21. Gong JP (2010) Why are double network hydrogels so tough? Soft Matter 6(12):25832590.

22. Joshi A, et al. (2006) Functional compressive mechanics of a PVA/PVP nucleus pulposus replacement. Biomaterials 27(2):176-184.

23. Haque MA, Kurokawa T, Kamita G, \& Gong JP (2011) Lamellar bilayers as reversible sacrificial bonds to toughen hydrogel: hysteresis, self-recovery, fatigue resistance, and crack blunting. Macromolecules 44(22):8916-8924.

24. Bai T, et al. (2011) Construction of an ultrahigh strength hydrogel with excellent fatigue resistance based on strong dipole-dipole interaction. Soft Matter 7(6):2825-2831.

25. Yu Q, Bauer JM, Moore JS, \& Beebe DJ (2001) Responsive biomimetic hydrogel valve for microfluidics. Applied Physics Letters 78(17):2589-2591.

26. Suresh S (1998) Fatigue of materials (Cambridge university press).

27. Suresh S \& Ritchie RO (1984) Propagation of short fatigue cracks. International Metals Reviews 29(1):445-475. 
28. Canale LdCF, Totten GE, \& Mesquita RA (2008) Failure analysis of heat treated steel components (ASM international).

29. Rivlin R \& Thomas A (1953) Rupture of rubber. I. Characteristic energy for tearing. Journal of Polymer Science 10(3):291-318.

30. Thomas A (1958) Rupture of rubber. V. Cut growth in natural rubber vulcanizates. Journal of Polymer Science 31(123):467-480.

31. Lake G \& Thomas A (1967) The strength of highly elastic materials. Proceedings of the Royal Society of London A: Mathematical, Physical and Engineering Sciences, (The Royal Society), pp 108-119.

32. Mars W \& Fatemi A (2002) A literature survey on fatigue analysis approaches for rubber. International Journal of Fatigue 24(9):949-961.

33. Mars W \& Fatemi A (2004) Factors that affect the fatigue life of rubber: a literature survey. Rubber Chemistry and Technology 77(3):391-412.

34. Lake G \& Lindley P (1965) The mechanical fatigue limit for rubber. Journal of Applied Polymer Science 9(4):1233-1251.

35. Tang J, Li J, Vlassak JJ, \& Suo Z (2017) Fatigue fracture of hydrogels. Extreme Mechanics Letters 10:24-31.

36. Yang $\mathrm{CH}$, et al. (2013) Strengthening alginate/polyacrylamide hydrogels using various multivalent cations. ACS applied materials \& interfaces 5(21):10418-10422.

37. Mullins L (1948) Effect of stretching on the properties of rubber. Rubber Chemistry and Technology 21(2):281-300.

38. Mullins L (1969) Softening of rubber by deformation. Rubber chemistry and technology 42(1):339-362.

39. Gent A (1996) Adhesion and strength of viscoelastic solids. Is there a relationship between adhesion and bulk properties? Langmuir 12(19):4492-4496.

40. Zhao X, Huebsch N, Mooney DJ, \& Suo Z (2010) Stress-relaxation behavior in gels with ionic and covalent crosslinks. Journal of Applied Physics 107(6):063509.

41. Creton C \& Ciccotti M (2016) Fracture and adhesion of soft materials: a review. Reports on Progress in Physics 79(4):046601.

42. $\mathrm{Hu} \mathrm{Y} \&$ Suo Z (2012) Viscoelasticity and poroelasticity in elastomeric gels. Acta Mechanica Solida Sinica 25(5):441-458.

43. Long R \& Hui C-Y (2016) Fracture toughness of hydrogels: measurement and interpretation. Soft Matter 12(39):8069-8086.

44. Hong W, Zhao X, Zhou J, \& Suo Z (2008) A theory of coupled diffusion and large deformation in polymeric gels. Journal of the Mechanics and Physics of Solids 56(5):1779-1793.

45. $\mathrm{Hu} \mathrm{Y}$, Zhao X, Vlassak JJ, \& Suo Z (2010) Using indentation to characterize the poroelasticity of gels. Applied Physics Letters 96(12):121904.

46. Kalcioglu ZI, Mahmoodian R, Hu Y, Suo Z, \& Van Vliet KJ (2012) From macro-to microscale poroelastic characterization of polymeric hydrogels via indentation. Soft Matter 8(12):3393-3398.

47. Baumberger T, Caroli C, \& Martina D (2006) Solvent control of crack dynamics in a reversible hydrogel. Nature materials 5(7):552-555.

48. Brown HR (2007) A model of the fracture of double network gels. Macromolecules 40(10):3815-3818. 
49. Tanaka Y (2007) A local damage model for anomalous high toughness of doublenetwork gels. EPL (Europhysics Letters) 78(5):56005.

50. Webber RE, Creton C, Brown HR, \& Gong JP (2007) Large strain hysteresis and mullins effect of tough double-network hydrogels. Macromolecules 40(8):2919-2927.

51. Wang X \& Hong W (2011) Pseudo-elasticity of a double network gel. Soft Matter 7(18):8576-8581.

52. Zhao X (2012) A theory for large deformation and damage of interpenetrating polymer networks. Journal of the Mechanics and Physics of Solids 60(2):319-332.

53. Zhang T, Lin S, Yuk H, \& Zhao X (2015) Predicting fracture energies and crack-tip fields of soft tough materials. Extreme Mechanics Letters 4:1-8.

54. Lu T, Wang J, Yang R, \& Wang T (2016) A constitutive model for soft materials incorporating viscoelasticity and Mullins effect. Journal of Applied Mechanics.

55. Yu QM, Tanaka Y, Furukawa H, Kurokawa T, \& Gong JP (2009) Direct observation of damage zone around crack tips in double-network gels. Macromolecules 42(12):38523855.

56. Gent AN (2012) Engineering with rubber: how to design rubber components (Carl Hanser Verlag GmbH Co KG).

57. Gent A \& Lai SM (1994) Interfacial bonding, energy dissipation, and adhesion. Journal of Polymer Science Part B: Polymer Physics 32(8):1543-1555. 